\title{
An Adaptive GTS Allocation Scheme for IEEE 802.15.4
}

\author{
Yu-Kai Huang, Student Member, IEEE, Ai-Chun Pang, Member, IEEE, and Hui-Nien Hung
}

\begin{abstract}
IEEE 802.15 .4 is a new standard uniquely designed for low-rate wireless personal area networks. It targets ultralow complexity, cost, and power for low-rate wireless connectivity among inexpensive, portable, and moving devices. IEEE 802.15.4 provides a Guaranteed Time Slot (GTS) mechanism to allocate a specific duration within a superframe for time-critical transmissions. This paper proposes an adaptive GTS allocation (AGA) scheme for IEEE 802.15.4, which considers low latency and fairness. The scheme is designed based on the existing IEEE 802.15.4 medium access control protocol, and IEEE 802.15.4 devices can receive this AGA service without any modification. A simulation model and an analytical model are developed to investigate the performance of our AGA scheme. The numerical results show that the proposed scheme significantly outperforms the existing IEEE 802.15.4 implementation.
\end{abstract}

Index Terms-IEEE 802.15.4, Zigbee, GTS, bandwidth allocation, fairness.

\section{INTRODUCTION}

W ITH the success of wireless local area networks, the wireless networking community has been looking for new avenues to extend wireless connectivity to existing and new applications [7].The emergence of short-transmissionrange wireless devices further boosts the development of wireless personal area networks (WPANs). A WPAN is a wireless network for device interconnection focused on an individual workspace. Among the well-known WPAN specifications, ultrawideband (that is, IEEE 802.15.3) is designed for high-rate WPANs [2]. Bluetooth (that is, IEEE 802.15.1) supports various applications such as wireless headsets of home audio and computer peripherals and provides quality-of-service (QoS) transmissions, especially for audio traffic [17]. When low cost and low power consumption are considered, Zigbee (that is, IEEE 802.15.4) emerges as a good alternative WPAN [3].

IEEE 802.15.4 targets ultralow complexity, cost, and power for low-rate wireless connectivity among inexpensive portable moving devices [4]. Such a WPAN might consist of multiple traffic types, including periodic data, intermittent data, and repetitive low-latency data [1]. In order to support time-critical data transfers generated by repetitive low-latency applications, IEEE 802.15.4 provides a Guaranteed Time Slot (GTS) mechanism to allocate a specific duration within a superframe for data transmissions. Although the dedicated bandwidth could guarantee the reliability and performance of data deliveries, the abuse of dedicated resources might result in the exclusion of other

- Y.-K. Huang and A.-C. Pang are with the Graduate Institute of Networking and Multimedia, Department of Computer Science and Information Engineering, National Taiwan University, Taipei 10617, Taiwan, R.O.C. E-mail: \{d94944009, acpang\}@csie.ntu.edu.tw.

- H.-N. Hung is with the Institute of Statistics, National Chiao Tung University, Hsinchu 30010, Taiwan, R.O.C.

E-mail:hhung@stat.nctu.edu.tw.

Manuscript received 15 Nov. 2006; revised 19 Apr. 2007; accepted 8 Aug. 2007; published online 5 Sept. 2007.

Recommended for acceptance by Y. Pan.

For information on obtaining reprints of this article, please send e-mail to: tpds@computer.org, and reference IEEECS Log Number TPDS-0364-1106. Digital Object Identifier no. 10.1109/TPDS.2007.70769. transmissions. This issue is further complicated by the firstcome, first-served (FCFS) GTS allocation policy [3] because of the scheduling inflexibility in low-latency data delivery in responding to network workload and to application needs. Starvation is even possible for devices with low data transmission frequencies due to a fixed timer maintained in IEEE 802.15.4 for GTS deallocation.

Based on the IEEE 802.15.4 specifications, the performance of IEEE 802.15.4 has been extensively investigated [13], [18]. Specifically, the impact of Zigbee network topologies on latency and energy consumption were studied in [19] and [14]. Performance analysis for an IEEE 802.15.4 beacon-enabled cluster with downlink and uplink traffic was presented in [15]. Koubaa et al. proposed an implicit GTS allocation mechanism (i-GAME) to improve the GTS utilization efficiency [9]. Furthermore, existing TDMA-based scheduling mechanisms [20], [12] for GPRS and WiMax networks are improper for the GTS allocation of IEEE 802.15.4. With sufficient resources, GPRS and WiMax networks support a variety of applications that require different QoS attributes, which makes their scheduling algorithms much more complicated than that for the IEEE 802.15.4 GTS allocation.

This paper proposes an adaptive GTS allocation (AGA) scheme for IEEE 802.15.4, considering low latency and fairness. There are two phases for the proposed scheme. In the classification phase, devices are assigned priorities in a dynamic fashion based on recent GTS usage feedbacks. Devices that need more attention from the coordinator are given higher priorities. In the GTS scheduling phase, GTSs are given to devices in a nondecreasing order of their priorities. A starvation avoidance mechanism is presented to regain service attention for lower priority devices that need more GTSs for data transmissions. The proposed AGA scheme is developed based on the standard of the IEEE 802.15.4 medium access control (MAC) protocol and completely follows the specification defined in [3] without introducing any extra protocol overhead. An analytical model and a simulation model are developed for our AGA scheme, where practical traffic models such as Gamma and Pareto distributions are 


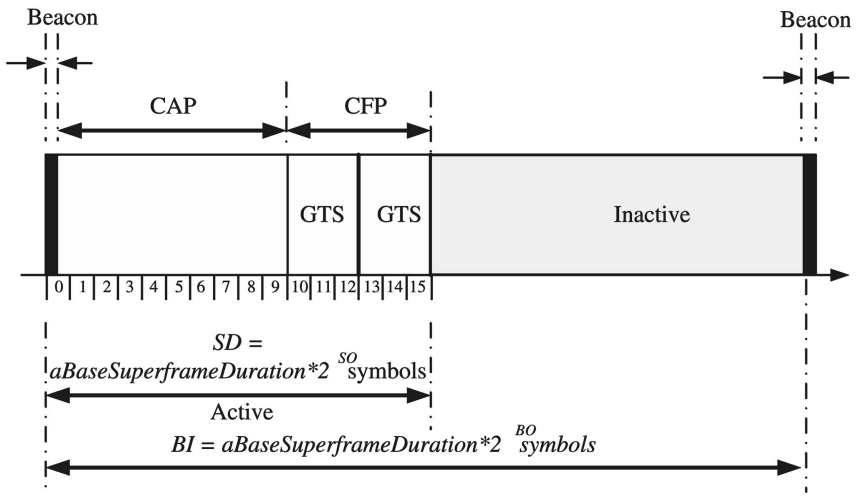

Fig. 1. Superframe structure in IEEE 802.15.4.

adopted. Based on the models, a series of experiments are conducted to show the capability of our AGA.

The rest of this paper is organized as follows: Section 2 describes the MAC protocol for IEEE 802.15.4. Section 3 defines the problem under investigation and proposes an AGA algorithm to provide low latency and fair transmissions for IEEE 802.15.4. In Section 4, an analytical model for our AGA scheme is developed, and a series of experiments are conducted to investigate the performance of our AGA scheme. Section 5 is the conclusion.

\section{IEEE 802.15.4 Medium Access Control}

The IEEE 802.15.4 Standard defines the physical layer and MAC sublayer specifications for low-rate WPANs (LRWPANs) [3]. The Standard supports wireless communications between devices with minimal power consumption and typically operates in a personal operating space of $10 \mathrm{~m}$ or less. The IEEE 802.15.4 defines two medium access modes: the nonbeacon-enabled mode and the beaconenabled mode. In the nonbeacon-enabled mode, arbitration of medium accesses is purely distributed among wireless devices based on Carrier Sense Multiple Access/Collision Avoidance (CSMA/CA). In addition to CSMA/CA-based transmissions, the beacon-enabled mode provides a contention-free GTS mechanism to support time-critical data deliveries. This paper focuses on the IEEE 802.15.4 beaconenabled mode, whereas the details for the nonbeaconenabled mode can be found in [3].

Fig. 1 shows a superframe structure adopted by the IEEE 802.15.4 beacon-enabled mode. A superframe begins with a beacon issued by a PAN coordinator and consists of an active portion and an inactive portion. The duration (also called the beacon interval (BI)) of a superframe ranges from $15 \mathrm{~ms}$ to 245 seconds. The coordinator and devices can communicate with each other during the active period and enter the low-power mode during the inactive period. The parameter macBeaconOrder $(B O)$ determines the length of the $B I$ (that is, $B I=2^{B O} \times$ aBaseSuperFrameDuration), and the parameter macSuper FrameOrder $(S O)$ decides the length of an active period $\left(S D=2^{S O} \times\right.$ aBaseSuperFrame Duration) in a superframe. The active portion with 16 time slots is composed of three parts: a beacon, a contention access period $(\mathrm{CAP})$, and a contention-free period (CFP). The beacon is transmitted by the coordinator at the start of slot 0 , and the CAP follows immediately after the beacon. In the CAP, a slotted CSMA/CA mechanism is used for devices to access the channel. In addition to nontime-critical data frames,
MAC commands such as association requests and GTS requests are transmitted in the CAP.

The IEEE 802.15.4 Standard defines the use of CFP for devices requiring dedicated bandwidth. The PAN coordinator is responsible for the GTS allocation and determines the length of the CFP in a superframe. Basically, the CFP length depends on the GTS requests and the current available capacity in the superframe. Provided that there is sufficient capacity in a superframe, the maximum number of GTS that the PAN coordinator can allocate is seven. The GTS direction relative to the data flow from the device that owns the GTS is specified as either transmit or receive. The transmit GTSs are used for transmitting data from devices to the PAN coordinator, and the downlink frames from the PAN coordinator to devices are delivered over the receive GTSs.

The device that requests new GTS allocation sends a GTS request command to the PAN coordinator during the CAP. Upon receipt of the GTS request command, the PAN coordinator first checks if there is available capacity in the current superframe. Provided that there is sufficient bandwidth in the current superframe, the coordinator determines, based on an FCFS fashion, a device list for GTS allocation in the next superframe. Then, the PAN coordinator includes the GTS descriptor (that is, the device list that obtains GTSs) in the following beacon to announce the allocation information. For GTS deallocation, devices can return the GTS resources by explicitly requesting that the PAN coordinator provide deallocation. However, in most cases, the PAN coordinator has to detect the activities of the devices occupying GTSs and determine when the devices stop using their GTSs. In IEEE 802.15.4, a fixed expiration timer is used to manage the GTS usage. Once the allocated GTSs is not utilized for $2^{i}$ superframes, the PAN coordinator reclaims the previously allocated GTS resources for those devices, where $i$ is defined as follows:

$$
\begin{cases}i=2^{8-B O}, & 0 \leq B O \leq 8 \\ i=1, & 9 \leq B O \leq 14 .\end{cases}
$$

\section{An Adaptive Guaranteed Time Slot ALLOCATION SCHEME}

The objective of this section is to propose an AGA scheme for IEEE-802.15.4-based WPANs, which considers low latency and fairness. In IEEE 802.15.4, GTS is provided by a PAN coordinator in a star network topology (see Fig. 2). 


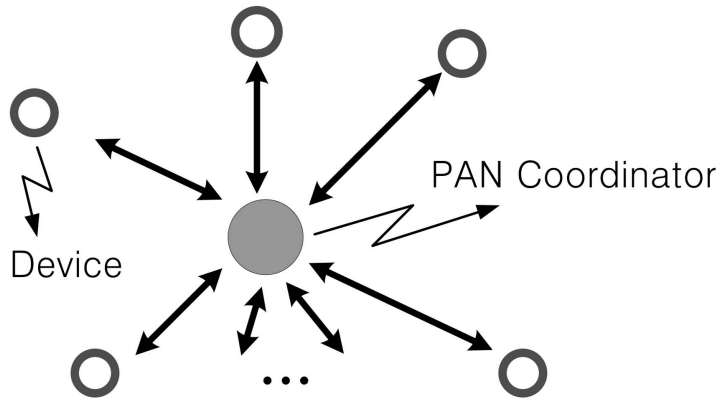

Fig. 2. Star topology in WPAN.

The PAN coordinator can communicate with up to 255 devices and update its GTS descriptor to the devices by periodically broadcasting a beacon frame.

An ideal GTS allocation scheme has a good estimate of the future GTS usage behaviors of devices. With the estimate, the PAN coordinator allocates GTS resources to needy devices and reclaims the previously allocated but unused GTSs. To achieve this goal, our AGA scheme adopts a two-phase approach. In the classification phase, devices are assigned priorities in a dynamic fashion based on recent GTS usage feedbacks. Devices that need more attention from the coordinator are given higher priorities. In the GTS scheduling phase, GTSs are given to devices in a nondecreasing order of their priorities. A starvation avoidance mechanism is presented to regain service attention for lower priority devices. Before presenting the details of the device classification and GTS scheduling phases, we define GTS hit and GTS miss as follows:

Definition 1. If one device has issued a successful GTS request in the CAP or transmitted data within its allocated GTS to the $P A N$ coordinator during the period of the current superframe, the device is defined to have a GTS hit. Otherwise, the device is considered to have a GTS miss.

\subsection{Device Classification Phase}

In the device classification phase, each device is adaptively classified into one state and is dynamically assigned a priority number by the coordinator based on its past GTS usage feedback. Assume that there are $N$ devices in an IEEE-802.15.4-based WPAN and that there are
$K+1(0,1, \cdots, K)$ priority numbers dynamically assigned to the $N$ devices. A large priority number represents a low priority for GTS allocation. The priority number assigned to the device $n$ is defined as $P_{r_{n}}$, and then, we have $0 \leq P_{r_{n}} \leq K$. In the AGA scheme, the devices with higher priorities are assumed to have more recent traffic and thus have higher probabilities to transmit their data in the subsequent superframe. The state and the priority number of a device are internally maintained by the PAN coordinator. The maintenance of the state and the priority number of each device is based on the concepts of Dynamic Branch Prediction for computer architecture designs [16] and of the Additive-Increase/Multiplicative-Decrease (AIMD) algorithm for network congestion control [10] but with some improvement. The details of the state and priority maintenance are described as follows:

\subsubsection{State Transition}

As shown in Fig. 3, all devices are classified into four traffic levels according to the state diagram. In this figure, the four traffic levels of devices are accordingly mapped to the four states, that is, Very High $(V H)$, High $(H)$, Middle $(M)$, and Low $(L)$, and the order of traffic levels for these states is $V H>H>M>L$. Initially, all devices are placed in the $L$ state. At the end of each superframe, the PAN coordinator examines the GTS usage of all devices and then decides the next states to which every device transits. The transition follows the solid and dashed lines in Fig. 3, and those lines respectively represent the occurrence of a GTS hit or a GTS miss. With the state diagram, the devices with more frequent GTS usage have larger probabilities to stay in heavytraffic states (for example, $V H$ and $H$ ). In addition, temporarily unstable transmission behaviors of devices are more tolerated. For example, the devices residing in the $\mathrm{VH}$ state with an occasional transmission interruption have a second chance before being downgraded to the lower traffic level states. On the other hand, the devices in the $L$ state are promoted to the heavy-traffic states by having consecutive GTS hits.

In the original IEEE 802.15.4 Specification [3], devices intending to utilize GTSs for data transmission wait for the expiration of GTSs (that is, the allocated GTSs that have not been used for a specific period). This passive deallocation scheme for GTS resources may result in starvation of light-traffic devices. In contrast, by using the

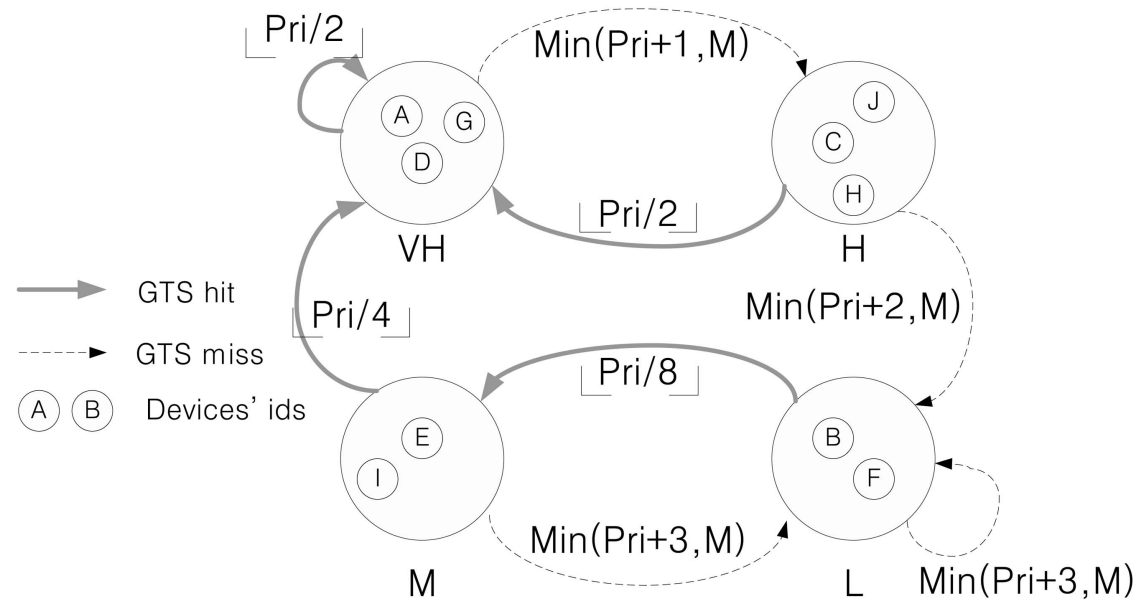

Fig. 3. State Diagram for Our AGA Scheme. 
new AGA scheme, starvation of light-traffic devices is avoided, since these devices are gradually promoted to the heavy-traffic state with the existing GTS-request facility to notify the PAN coordinator for traffic-level promotion.

\subsubsection{Priority Assignment}

By using the state diagram in Fig. 3, the PAN coordinator can monitor the recent transmission behaviors of devices and can classify the devices into proper traffic types. However, with scarce GTS resources (that is, seven time slots) of IEEE-802.15.4-based networks, the four-state classification of devices is somewhat rough. Thus, the state diagram in Fig. 3 is further revised so that each device is dynamically assigned a priority number for GTS allocation. By combining priority number assignment with the fourstate classification in the AGA scheme, the short-term transmission behaviors of a device can be monitored based on the state maintenance, whereas the priority number of a device reflects its long-term transmission characteristics. ${ }^{1}$

Upon the occurrence of a GTS hit on a device, the priority number of the device is decreased by the PAN coordinator, and the priority of GTS allocation for the device upgrades. On the other hand, when a GTS miss occurs at a device, the PAN coordinator increases the priority number of the device and, hence, the opportunity for obtaining a GTS for the device reduces. Maintenance of the priority numbers of devices depends on the transmission feedback and the traffic-level states of the devices, and the details are presented below.

Compared to the priority assignment by purely using AIMD [6], this scheme provides a multilevel AIMD algorithm for updating the priority numbers. In our multilevel priority updating, the decrease/increase of a priority number of a device depends on the traffic-level state of the device. The high-traffic-level devices with temporary interruption of GTS usage are slightly demoted to lower priorities. On the other hand, if a low-traffic-level device successfully issues a GTS request, its priority is greatly promoted to receive GTS service as soon as possible. Thus, starvation of such a low-priority device can be avoided. This priority assignment focuses on whether devices have continuous data to be transmitted over the GTSs. The devices with consecutive transmissions are favored by our scheme, and a device that is idle for a period of time is considered as needing the GTS service. Hence, greatly degrading the device's priority is reasonable.

In Fig. 3, we can see that if the device $n$ in the $V H$ state uses the GTS service all the time and occasionally has a GTS miss, its priority $P_{r_{n}}$ will be increased by 1 . Once the device $n$ resumes requesting for the GTS service in the following superframe and then has continuous data to be transmitted, the priority number for the device $n$ will be exponentially halved. For the device $k$ in the $L$ state, a similar and even greater priority promotion occurs if the device $k$ has consecutive GTS hits. On the other hand, if the device $k$ in the $L$ state just has one GTS hit and ceases transmitting data, the degradation of the priority for device $k$ would be more serious than that for the high-traffic-level device $n$.

\subsection{Guaranteed Time Slot Scheduling Phase}

With the device classification phase, priorities for GTS allocation for all devices, under the supervision of

1. A device with more recent transmissions will reside in a higher traffic state, even if its average transmission rate is pretty low. However, it has a large priority number. the PAN coordinator, are determined. Next, in the GTS scheduling phase, the GTS resources are adequately scheduled and allocated to the devices. The scheduling criteria are based on the priority numbers, the superframe length (depending on the $B O$ value), and the GTS capacity of the superframe. The GTS scheduling algorithm is shown in Procedure 1. Assume that there are $N$ devices in the WPAN and $P$ is a set of the priority numbers of the $N$ devices. In Procedure 1, the PAN coordinator first checks if the GTS capacity is overloaded. In the IEEE 802.15.4 Specification [3], the GTS capacity in a superframe shall meet the following requirements:

1. The maximum number of GTS slots to be allocated to devices is seven.

2. The minimum length of a CAP shall be aMinCAPLength. The increase in the total GTS period shall not result in the reduction of the CAP length to less than aMinCAPLength.

If the requirements are met, the GTS capacity is not considered overloaded. Provided that there are sufficient GTS resources to accommodate more devices, lines 5-11 of the WHILE loop are executed. At each iteration of the WHILE loop, a minimum $P_{r_{k}}$ among $P$ is selected, and this value is compared with a threshold value $T_{h}$. $T_{h}$ is defined as

$$
T_{h}=K R^{B O},
$$

where $\mathrm{R}$ is a constant, and $0<R \leq 1$. If $P_{r_{k}} \leq T_{h}$ (line 6), then the device $k$ is scheduled in the GTS of the current superframe.

The threshold $T_{h}$ is presented here due to the consideration of the CFP and CAP traffic loads. When the CFP traffic load is light (that is, most of the devices have high priority numbers), there is no need to allocate too many GTS resources for the devices. Too much dedicated bandwidth for GTS usage leads to resource wastage and to the degradation of the overall system performance. Instead, the GTS bandwidth should be transferred for contention-based accesses in CAP. By using the threshold $T_{h}$, the PAN coordinator filters unnecessary GTS allocation. The value of $T_{h}$ is dynamically adjusted and depends on the maximum priority number $K$, a constant $R$, and the $\mathrm{BI}$ determined from $B O$. As the $\mathrm{BI}$ increases, there is a higher probability that many devices have requested the GTS service in the superframe. Based on our priority assignment, the devices requesting GTS are assigned small priority numbers, even though they only have one request in the whole superframe. To prevent the scarce GTS resources from distributing to those devices with extremely low frequency GTS requests in such a long superframe, a more strict threshold is needed. In this case, the $T_{h}$ value is set to be much smaller than $K$. On the other hand, in a short BI, the value of $T_{h}$ can be increased, and the limitation for the device selection can be relaxed. When $R=1$, we have $T_{h}=K$; that is, no filtering action is triggered.

\section{Procedure 1: DEVICE SCHEDULING()}

1: Assume that there are $\mathrm{N}$ devices in the WPAN

2: $P=\left\{P_{r_{1}}, P_{r_{2}}, \cdots, P_{r_{N}}\right\}$

3: $T_{h}=K R^{B O}$, where $R$ is a constant

4: while The GTS capacity is not overloaded do

5: Find a device $k$ such that $P_{r_{k}} \in P$ is the minimum number of $P$ 
6: if $P_{r_{k}} \leq T_{h}$ then

7: $\quad$ The device $k$ will be scheduled in the GTS of the current superframe

8: $\quad$ Remove $P_{r_{k}}$ from $P$

9: else

10: break

11: end if

12: end while

\subsection{Discussions of the Adaptive Guaranteed Time Slot Allocation Implementation}

Here, we discuss some implementation issues based on our AGA scheme and the original IEEE 802.15.4 implementation:

- Loss of GTS requests. In IEEE 802.15.4, devices inform the coordinator of their need for GTS resources by issuing GTS requests in CAP. With contention-based transmissions of CAP, loss of GTS requests resulting from data collisions/congestions and signal interruption may occur, which leads to the delay of the resource grant from the coordinator. The impacts of GTS request loss on our AGA and the original IEEE 802.15.4 implementations are explained as follows:

When the traffic load in CAP is not heavy, request loss occasionally occurs mainly due to signal interruption. In most cases, the lost requests will be successfully transmitted to the coordinator in their subsequent superframe, which has little impact on the performance of both AGA and the original IEEE 802.15.4 implementations. However, for the case that GTS resources are almost fully occupied, this random loss for the original IEEE 802.15.4 implementation could result in long waiting times for the lost requests to be granted to GTS resources. The negative phenomenon comes from the IEEE 802.15.4 FCFS allocation and from a fixedtimer deallocation policy. Even if the request $R_{i}$ lost in the current superframe is successfully delivered to the coordinator in the subsequent superframe, the GTS resources have been allocated to a later successful request $R_{j}$ and will be kept by $R_{j}$ for a long time. On the other hand, a single loss for a request $R_{i}$ (that is, GTS miss) in our AGA will be more tolerated. The priority of $R_{i}$ can be quickly recovered, as long as $R_{i}$ is successfully delivered to the coordinator in the subsequent superframe.

As the CAP traffic load increases and data collisions/congestions frequently occur, GTS requests are rarely delivered to the coordinator. Both our AGA and the original IEEE 802.15.4 implementations suffer from this problem, and their waiting times for GTS resources are significantly lengthened. However, this problem could be slightly relaxed by our AGA through the dynamic adjustment of the value of the threshold $T_{h}$. Decreasing the value of $T_{h}$ will shorten the duration of CFP and simultaneously extend the length of CAP.

- Implementation overhead. To implement our AGA in the coordinator for adaptively allocating the GTS resources, some extra information for devices shall be recorded, as compared to the original IEEE 802.15.4 implementation. Specifically, the priority number (7 bits for 100 priority numbers) and state ( 2 bits for four states) for each device are maintained in the coordinator. With $N$ devices in an IEEE 802.15.4 network, the additional memory size for keeping the extra information for our AGA is $9 N$ bits (about $N$ bytes).

- Backward compatibility. Our AGA is fully backward compatible with the implementation of IEEE 802.15.4 devices. IEEE 802.15.4 Standard devices can receive our AGA service without any modification. Furthermore, the proposed AGA scheme is developed based on the standard of the IEEE 802.15.4 MAC protocol and completely follows both the message type/format and flow defined in the IEEE 802.15.4 Specifications. The manufacturers that intend to implement AGA for their IEEE-802.15.4-based products only need to replace the legacy GTS allocation/deallocation of the coordinator with our AGA scheme.

\section{Performance Evaluation}

This section develops an analytical model and a simulation model to investigate the performance of our AGA scheme. In our models, a star topology with $N$ devices surrounding one coordinator is assumed. Only the transmit GTSs for the uplink traffic are considered, and all GTS transmission are successful (that is, no retransmissions). Each device is allocated at most one GTS slot, and according to the IEEE 802.15.4 Specifications, the maximum GTS number $G_{n}$ in a superframe is 7 . The packet arrivals for each device form a Poisson stream with the interarrival rate $\lambda$, and each new arriving packet shall trigger the issuance of a GTS request in the superframe. If there are no sufficient GTS resources for the request, the device will reissue the request for the packet in the subsequent superframe.

Before describing our analytical model, we list the notations that will be used in the model (see Table 1).

\subsection{Analytical Modeling}

This section models our AGA scheme and derives the distribution of the priority number for a device in a superframe. The priority distribution $D$ of a device can be expressed as

$$
D=\left(\Pi_{0}, \Pi_{1}, \Pi_{2}, \cdots\right),
$$

where $\Pi_{n}$ denotes the stationary probability of the device with priority number $n$. Let $s$ denote the state where the device resides, where the $s$ values $1,2,3$, and 4 respectively represent the states $V H, H, M$, and $L$. Then, we have

$$
\Pi_{n}=\sum_{s=1}^{4} \pi_{(s, n)},
$$

where $\pi_{(s, n)}$ is the probability that the device resides at state $s$ and has the priority number $n$. Then, $\pi_{(s, n)}$ can be

$$
\pi_{(s, n)}=\sum_{\forall 1 \leq r \leq 4,0 \leq m \leq K} \pi_{(r, m)} P_{t_{(r, m),(s, n)}} .
$$

In (4), $P_{t_{(r, m),(s, n)}}$ is defined as the transition probability (from the current superframe to the subsequent superframe) that the device transits from state $r$ to state $s$, and its priority number is changed from $m$ to $n$. According to the priority assignment rule described in Section 3.1.2, $P_{t_{(r, m),(s, n)}}$ can be 
TABLE 1

Notations Used in the Analytical Model

\begin{tabular}{||c|l||}
\hline Notation & Description \\
\hline$\Pi_{n}$ & The stationary probability of the device with priority number $n$ \\
\hline$\pi_{(s, n)}$ & $\begin{array}{l}\text { The probability that the device resides at state } s \text { and has priority } \\
\text { number } n\end{array}$ \\
\hline$P_{t_{(r, m),(s, n)}}$ & $\begin{array}{l}\text { The transition probability that the device transits from state } r \text { to state } \\
s \text { and its priority number is changed from } m \text { to } n\end{array}$ \\
\hline$P_{a_{(r, m)}}$ & $\begin{array}{l}\text { The probability that the device with state } r \text { and priority number } m \\
\text { will be promoted to a high priority in the subsequent superframe }\end{array}$ \\
\hline$P_{g}$ & $\begin{array}{l}\text { The probability that the device generates a packet during the current } \\
\text { beacon interval }\end{array}$ \\
\hline$A(i, j, z, q)$ & $\begin{array}{l}\text { The probability that among all devices in the system, there are } i \\
\text { devices with their priority numbers larger than } q, j \text { devices with their } \\
\text { priority numbers less than } q \text {, and } z \text { devices with their priority numbers } \\
\text { equal to } q\end{array}$ \\
\hline$U_{q}$ & $\begin{array}{l}\text { The probability that the device assigned priority number } q \text { in the } \\
\text { previous superframe was not allocated GTS resources for the packet } \\
\text { arrival in the previous superframe }\end{array}$ \\
\hline
\end{tabular}

classified into two conditions. For the case of priority promotion of the device (that is, the solid lines in Fig. 3)

$$
\begin{aligned}
& P_{t_{(1, m),\left(1, n=\left\lfloor\frac{m}{2}\right\rfloor\right)}}=P_{a_{(1, m)},}, \\
& P_{t_{(2, m),\left(1, n=\left\lfloor\frac{m}{2}\right\rfloor\right)}}=P_{a_{(2, m)}}, \\
& P_{t_{(3, m),\left(1, n=\left\lfloor\frac{m}{4}\right\rfloor\right)}}=P_{a_{(3, m)},}, \\
& P_{t_{(4, m),\left(3, n=\left\lfloor\frac{m}{8}\right\rfloor\right)}}=P_{a_{(4, m)}},
\end{aligned}
$$

where $P_{a_{(r, m)}}$ is the probability that the device with state $r$ and priority number $m$ in the current superframe will be promoted to a high priority in the subsequent superframe. On the other hand, for the case of priority degradation (that is, the dashed lines in Fig. 3), $P_{t_{(r, m),(s, n)}}$ can be expressed as

$$
\begin{aligned}
& P_{t_{(1, m),(2, m+1)}}=1-P_{a_{(1, m)}}, \\
& P_{t_{(2, m),(4, m+2)}}=1-P_{a_{(2, m)}}, \\
& P_{t_{(3, m),(4, m+3)}}=1-P_{a_{(3, m)}}, \\
& P_{t_{(4, m),(4, m+3)}}=1-P_{a_{(4, m)}} .
\end{aligned}
$$

In our AGA scheme, whether a device with state $r$ and priority number $m$ will be promoted depends on the occurrence of the event of GTS hit in the current superframe. Thus, $P_{a_{(r, m)}}$ will be

$$
P_{a_{(r, m)}}=P_{g}+\left\{\frac{\left(1-P_{g}\right)\left[\sum_{\forall p, q} \pi_{(p, q)} P_{t_{(p, q),(r, m)}} U_{q}\right]}{\left[\sum_{\forall p, q} \pi_{(p, q)} P_{t_{(p, q)(r, m)}}\right]}\right\} .
$$

The equation $P_{a_{(r, m)}}$ listed above is composed of two parts. The first part $P_{g}$ represents the probability that the device generates a packet during the current $\mathrm{BI}(B)$. In this case, the device issues a GTS request for the new packet, and its priority will be promoted in the subsequent superframe. Since the packet arrivals are assumed to be a Poisson stream with rate $\lambda, P_{g}$ can be expressed as

$$
P_{g}=1-e^{-\lambda B} \text {. }
$$

On the other hand, the device could have a priority promotion in the second part, even though there is no packet arrival in the current superframe. In this case, the occurrence of the GTS hit event is triggered by the GTS request of the packet arriving in the previous superframe. With the insufficiency of GTS resources in the previous superframe, the device did not obtain a GTS slot in the previous superframe and reissues a GTS request in the current superframe.

$U_{q}$ represents the probability that the device assigned priority number $q$ in the previous superframe was not allocated GTS resources for the packet arrival in the previous superframe. Let $A(i, j, z, q)$ denote the probability that among all devices in the system, there are $i$ devices whose priority numbers are larger than $q, j$ devices whose priority numbers are less than $q$, and $z$ devices whose priority numbers are equal to $q$. Then, we have

$$
A(i, j, z, q)=\left(\sum_{k>q} \Pi_{k}\right)^{i}\left(\sum_{k<q} \Pi_{k}\right)^{j} \Pi_{q}^{z} .
$$

Based on the above equation, $U_{q}$ can be expressed as

$$
\begin{array}{r}
U_{q}=\sum_{i<N-G_{n}} \sum_{j<N-i}\left[\left(\begin{array}{c}
N-1 \\
i
\end{array}\right)\left(\begin{array}{c}
N-1-i \\
j
\end{array}\right)\right. \\
\left.\cdot A(i, j, N-1-I-j, q) P_{b}\right],
\end{array}
$$

where

$$
P_{b}= \begin{cases}\frac{N-G_{n}-i}{N-i-j}, & \text { if } \frac{N-G_{n}-i}{N-i-j}<1 \\ 1, & \text { otherwise. }\end{cases}
$$

Based on the self-consistent (2), (3), (4), (5), (6), (7), and (8), the priority number distribution $D$ for our AGA device can be calculated using the iteration methods. Fig. 4 shows the priority number distribution $D$ obtained from our mathematical analysis and simulation experiments, where $\lambda=0.3 / \mathrm{s}$. To show the applicability of our analytical model, the values of $N$ are set to 5 (less than the maximum GTS number) and 10 (greater than the maximum GTS number). Based on these figures, the results of our 


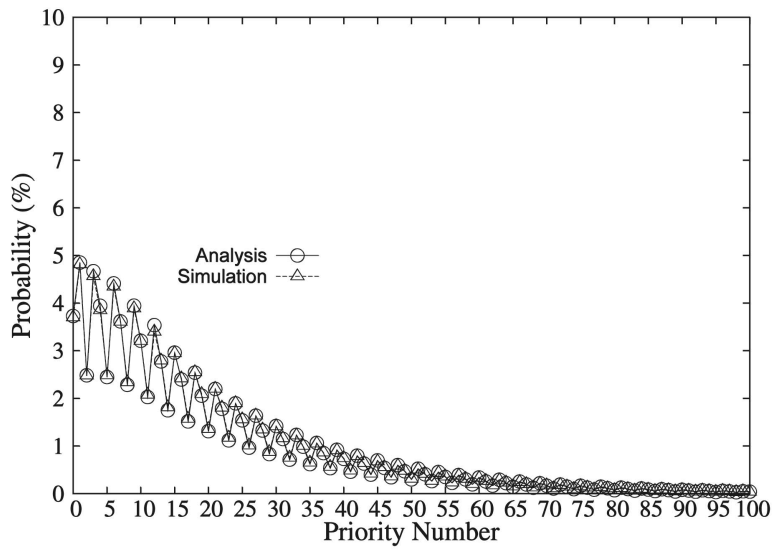

(a)

Fig. 4. Priority distribution $D$. (a) $N=5$. (b) $N=10$.

mathematical analysis closely match those of the simulation experiments. In addition, the fluctuation of the priority number distribution in these figures are shown due to the reason that our study is targeted at the GTS allocation for IEEE-802.15.4-based "low-rate" applications. With low-rate transmissions, devices probably have more "GTS miss" than "GTS hit" and reside in the "Middle" and "Low" states in the steady state. For our AGA scheme, the priority number is increased by 3 when the devices in the "Middle" and "Low" states incur "GTS miss." This phenomenon dominates the overall priority number updating; thus, the priority number distribution indicates a cycle of fluctuation every three priority numbers.

\subsection{Simulations and Numerical Results}

This section develops a simulation model to investigate the performance of our AGA scheme. Our developed simulation follows the specification of the IEEE 802.15.4 MAC protocol and is validated by our mathematical analysis. In the simulation model, a star topology with one PAN coordinator and $N$ devices $(N=10$ and 20$)$ is adopted. Each simulation run lasts 100,000 BIs (that is, 49,152 seconds). The packet arrivals for each device form a Poisson stream with the interarrival rate $\lambda$. In addition to Poisson arrivals, our simulation experiments are enhanced to accommodate Gamma and Pareto distributions for packet interarrival times to more practically evaluate the performance of our AGA scheme. The Gamma distribution is selected, because it can approximate many other distributions and experimental data [11], whereas the Pareto distribution can practically model Internet traffic [5]. In the experiments, we consider two Gamma distributions, and their shape parameters are respectively set to 0.5 and 2 (denoted as Gamma1 and Gamma2). The probability distribution of Gamma1 is similar to that of the Exponential distribution, whereas Gamma2 provides a near-Normal distribution.

Two traffic types generated by devices are considered: heavy traffic and light traffic. $\lambda_{h}$ and $\lambda_{l}$ represent respectively the interarrival rates for the heavy-traffic and light-traffic devices. In the simulations, we have $\lambda_{h}=0.3 / \mathrm{s}$ and $\lambda_{l}=0.1 / s$. Such rate settings are reasonable in IEEE-802.15.4-based WPANs, since IEEE 802.15.4 targets low-rate wireless communications. In addition, the ratio of the number of heavy-traffic devices to that of all devices is defined as $v$. Thus, the GTS traffic load $\Lambda$ will be

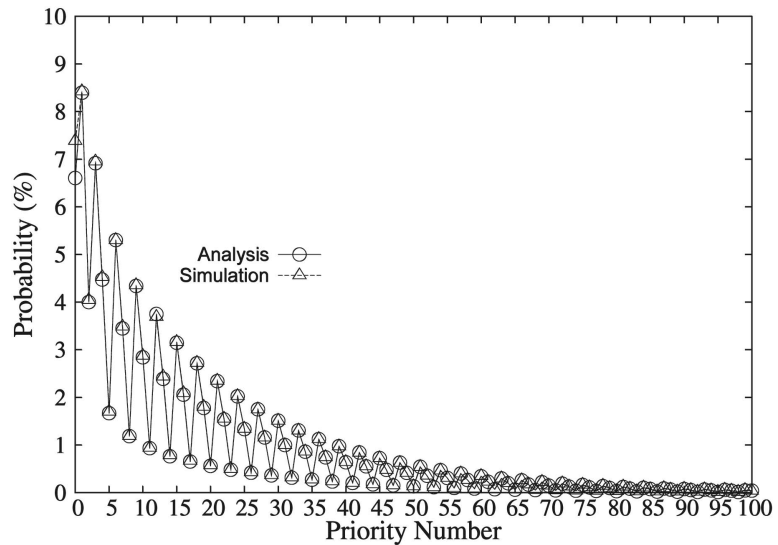

(b)

$N v \lambda_{h}+N(1-v) \lambda_{l}$. Table 2 lists the input parameters for our simulation model.

As to the output measures, the average packet waiting time is an important metric for our proposed AGA scheme. The deviation $\sigma$ of packet waiting times is also used to evaluate the performance of our AGA scheme. Furthermore, a fairness index $F$ for packet waiting times is utilized to measure the fairness among different traffic-type devices for our scheme. From [8], $F$ is defined as

$$
F=\frac{\left(\sum_{i=1}^{N} W_{i}\right)^{2}}{N \sum_{i=1}^{N} W_{i}^{2}},
$$

where $N$ is the total number of devices in the network, and $W_{i}$ is the average waiting time of packets generated by the device $i$. In (9), it is clear that $0 \leq F \leq 1$. When the average waiting times for all devices are close, the $F$ value approaches 1 . On the other hand, if the variation of the $W_{i}$ values becomes large, $F$ approaches to 0 . Therefore, a large $F$ implies that each device obtains the GTS bandwidth more fairly, and probably, starvation will not occur. Based on the input parameters and output measures, we use some numerical examples to evaluate the performance of our AGA scheme.

\subsubsection{Effects of $v$ on the Average Packet Waiting Time}

Fig. 5 shows the effect of the GTS traffic load $v$ on the average packet waiting time, where the dashed and solid lines respectively represent the original and our AGA schemes. In this figure, we have $N=10$ and 20 , and the

TABLE 2

System Parameters

\begin{tabular}{||c|c||}
\hline Parameters & value \\
\hline Frame Size & $127 \mathrm{~B}$ \\
\hline Transmission Rate & $250 \mathrm{kbps}$ \\
\hline Network Topology & Star topology \\
\hline Number of Devices & 10 and 20 \\
\hline$B O=S O$ & 5 \\
\hline$R$ & 1.0 \\
\hline Buffer Size of Each Device & 100 \\
\hline$\lambda_{h}$ & $0.3 / \mathrm{s}$ \\
\hline$\lambda_{l}$ & $0.1 / \mathrm{s}$ \\
\hline
\end{tabular}




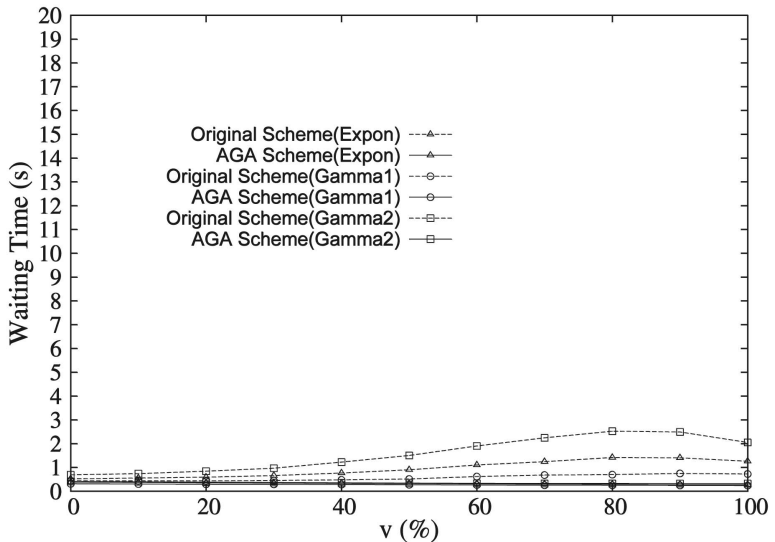

(a)

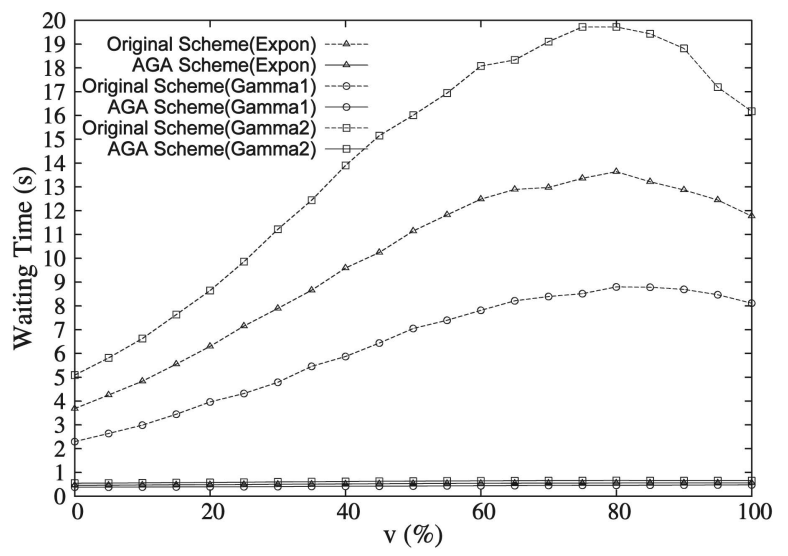

(c)

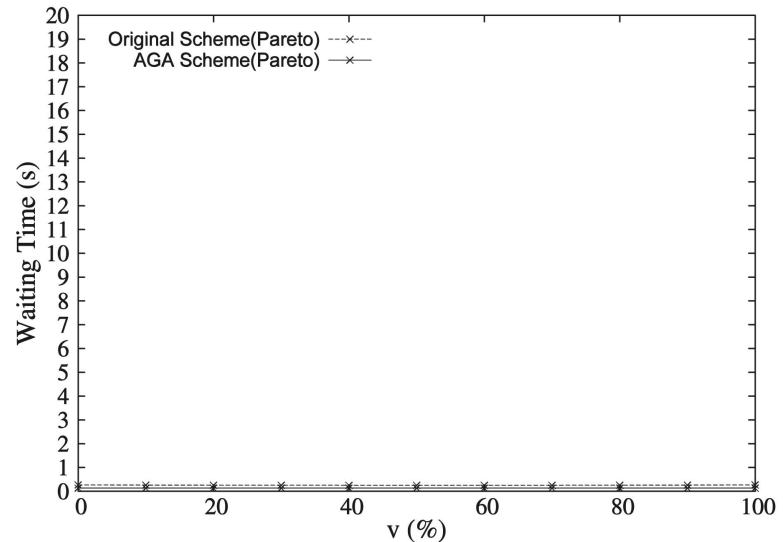

(b)

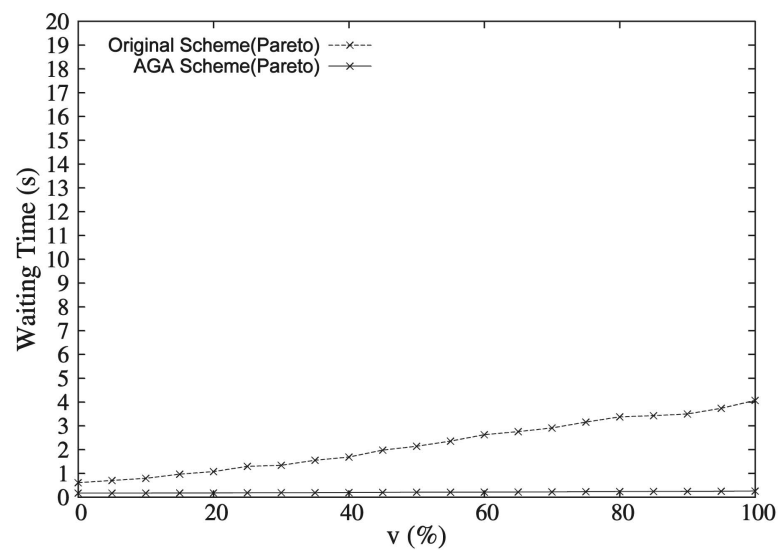

(d)

Fig. 5. Effect of traffic load on the average packet waiting time (in seconds). (a) $N=10$. (b) $N=10$. (c) $N=20$. (d) $N=20$.

interarrival times follow the Exponential, Gamma, and Pareto distributions. When $N=10$, the experimental results for the Exponential and Gamma distributed packet interarrival times are shown in Fig. 5a, whereas Fig. 5b indicates the results for the Pareto-distributed traffic. In Figs. 5a and $5 b$, we observe that our AGA scheme performs pretty well on the average packet waiting time for all $v$ and traffic models under investigation. Specifically, the average packet waiting times of our AGA scheme are independent of the traffic models, and its values remain small for all $v$.

However, the performance of the original scheme is sensitive to the traffic distribution of packet arrivals. When the packet interarrival times use the Gamma2 distribution (that is, the near-Normal distribution), the average packet waiting times for the original scheme will be larger than 2 seconds as $v \geq 60$ percent. With Gamma2, most of the packet interarrival times are generated around its mean value, and there is a considerable distinction of packet arrival frequency between heavy-traffic and light-traffic devices. Thus, the GTS resources will probably be occupied by the heavy-traffic devices, and the average packet waiting time is increased. Note that when the Pareto distribution is adopted, as shown in Fig. 5b, the average packet waiting time of the original scheme is not influenced by the GTS traffic load $v$. This phenomenon is due to the long-tailed characteristic of the Pareto distribution. With the Pareto traffic model, the GTS resources for the original scheme could be easily released by the devices that incur extremely long interarrival times generated by the Pareto distribution, and the starvation of light-traffic devices is not serious.

When the device number $N$ is increased to 20 (see Figs. 5c and $5 \mathrm{~d}$ ), the increasing rate of the latency for our AGA scheme is considerably smaller under all traffic distributions as the GTS traffic load $v$ increases. Our AGA provides more resistance to the increase in the traffic load, even if the network size is large. On the other hand, for the original IEEE 802.15.4 GTS allocation scheme, $v$ has a great impact on the latency as the network size is expanded. As $v$ increases, the average packet waiting times for the Gamma and Exponential traffic distributions significantly increase and then slightly decrease. The increase in latency results from the inflexibility of GTS allocation presented by the IEEE 802.15.4 Specifications. In this case, most of the GTS resources are occupied by heavy-traffic devices for a long time, which probably leads to the starvation (or near starvation) of lighttraffic devices. As $v$ becomes close to 1 , the number of starving devices with the light traffic load decreases, and thus, the average waiting time slightly decreases. Note that Fig. $5 \mathrm{~d}$ indicates that the average packet waiting time for the Pareto traffic distribution increases all the time as $v$ increases.

\subsubsection{Effects of $v$ on Fairness $F$}

Based on the index $F$, Fig. 6 shows the effect of the traffic load $v$ on the fairness of the GTS resource allocation for our AGA and the original schemes. In this figure, we observe that for most of the curves, $F$ decreases and then increases 


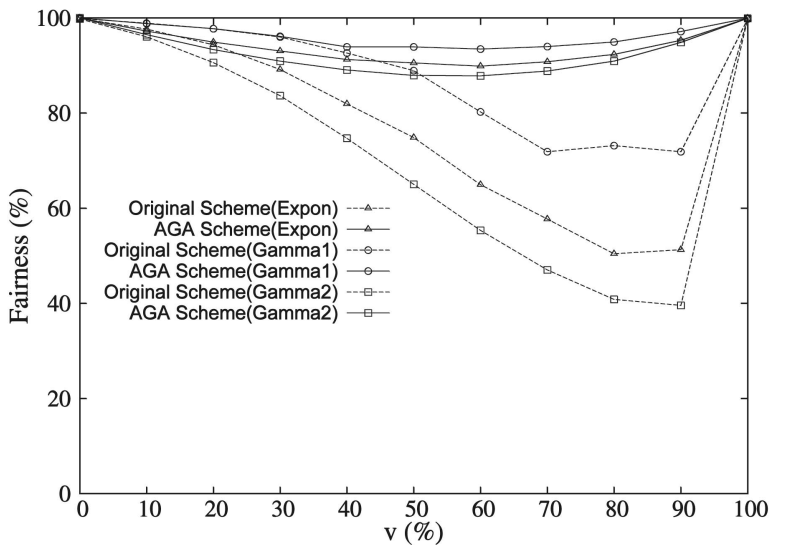

(a)

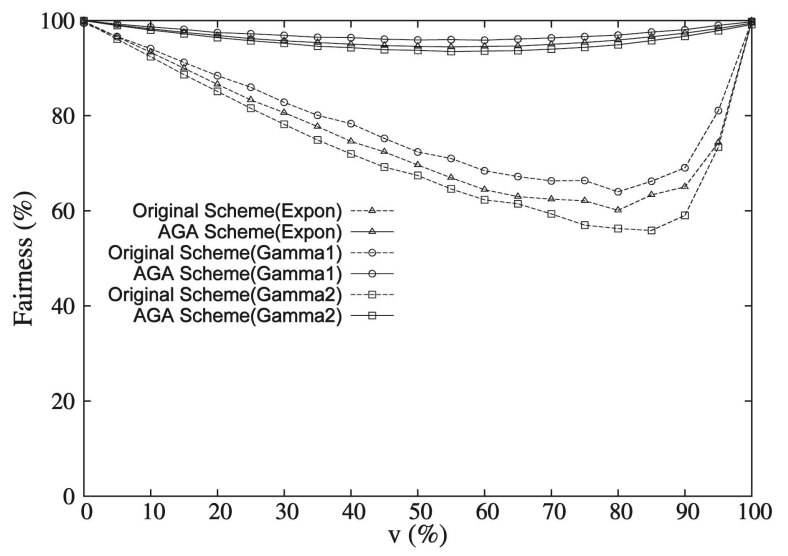

(c)

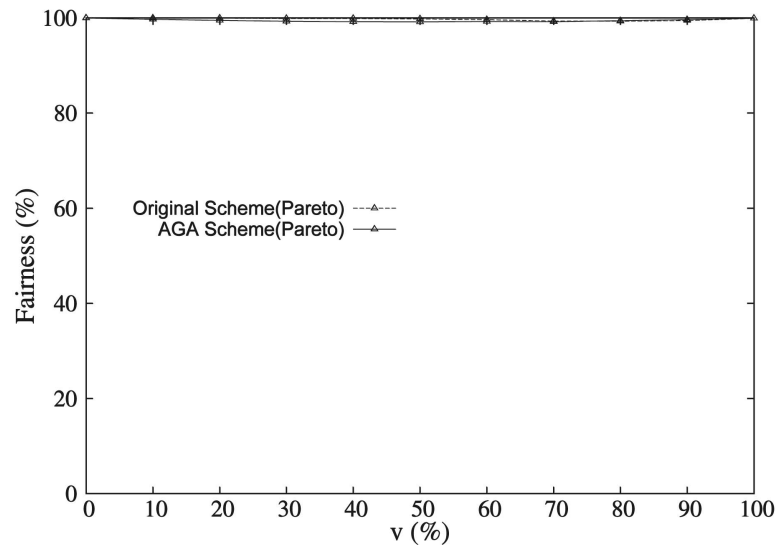

(b)

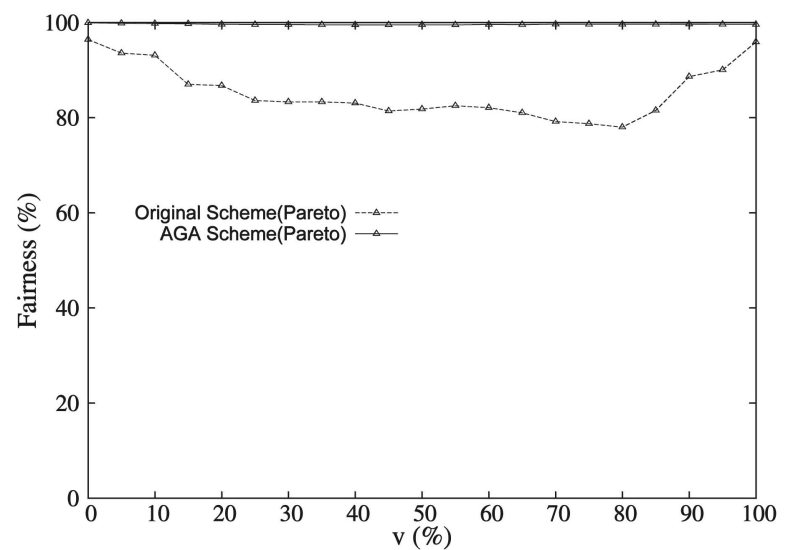

(d)

Fig. 6. Effect of traffic load on fairness index $F$. (a) $N=10$. (b) $N=10$. (c) $N=20$. (d) $N=20$.

as $v$ increases, which implies that the unfairness problem comes from the workload heterogeneity of devices. However, the decreasing/increasing rate of $F$ for our AGA is much smaller than that for the original scheme. That is, our proposed scheme is equipped with the capability to provide fair transmissions among different kinds of devices. Furthermore, we observe that when 70 percent $\leq v \leq$ 90 percent, a serious unfair situation occurs in the original IEEE 802.15.4 scheme, especially when the Gamma2 distribution is used for the traffic model. This implies that with a larger distinction of packet arrival frequency between heavy-traffic and light-traffic devices, more GTS resources are occupied by the heavy-traffic devices, and starvation of the light-traffic devices might exist.

Note that when $N=10$, both the original scheme and our AGA scheme performs well on fairness when the Pareto distribution is used. The reason is that most of the interarrival times under the Pareto distribution are close to zero, no matter what the traffic type (heavy traffic or light traffic) is. With similar interarrival times for heavy-traffic and light-traffic devices, the unfair situation seldom occurs.

\subsubsection{Effects of $v$ on the Standard Deviation of Packet Waiting Times}

Fig. 7 illustrates the standard deviation of packet waiting times for our AGA scheme and the original IEEE 802.15.4 scheme when the number $N$ of devices is equal to 10 and 20 . In this figure, the curves of the standard deviation for our
AGA scheme indicate that the variations of packet waiting times for GTS users are quite small, and their values are not affected by $v$. This phenomenon implies that our AGA can provide stable GTS transmissions and real-time data deliveries required by repetitive low-latency applications. On the other hand, the standard deviations of the original IEEE 802.15.4 scheme significantly increase and then slightly decrease as $v$ increases, and the values of these standard deviations are much larger than those of our AGA scheme. The increase in the standard deviation of the original scheme comes from the extremely unfair distribution of GTS resources. With the original GTS allocation scheme, the QoS requirements such as the delay and jitter of packet waiting times are rarely guaranteed.

\section{Conclusion}

To improve the performance of the GTS mechanism for IEEE 802.15.4 WPANs in the beacon-enabled mode, this paper presented a new GTS allocation scheme with dynamic resource allocation, which considers low latency and fairness. Our proposed scheme consists of two phases: the device-classification phase and the GTS-scheduling phase. In the device-classification phase, the priority is determined for each device intending to transmit data. Then, the GTS slots are adequately scheduled and allocated according to the priorities in the GTS scheduling phase. Our scheme was designed according to the existing IEEE 802.15.4 MAC protocol, and IEEE 802.15.4 devices 


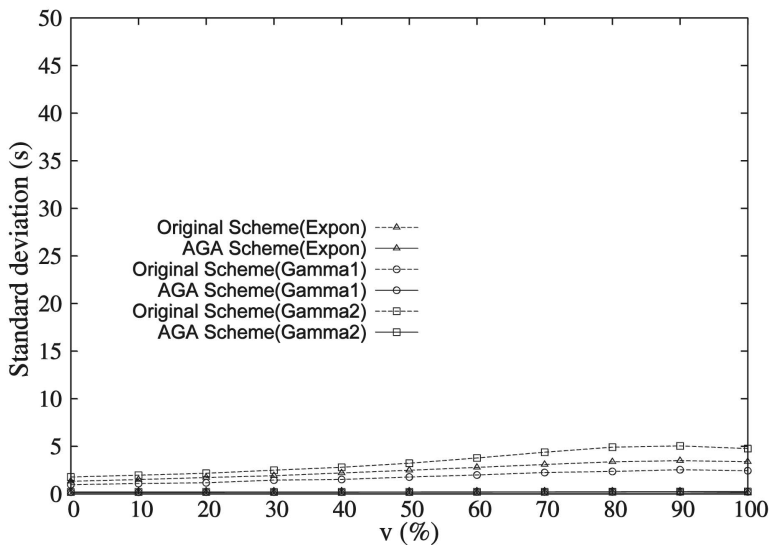

(a)

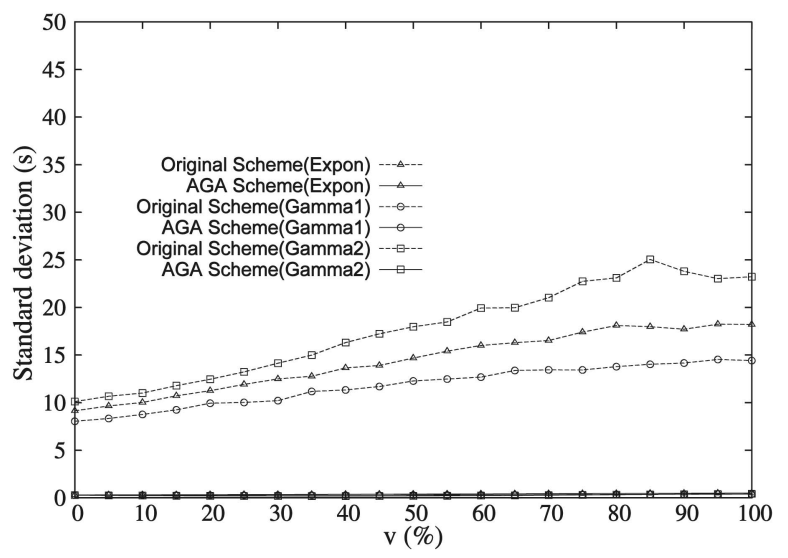

(c)

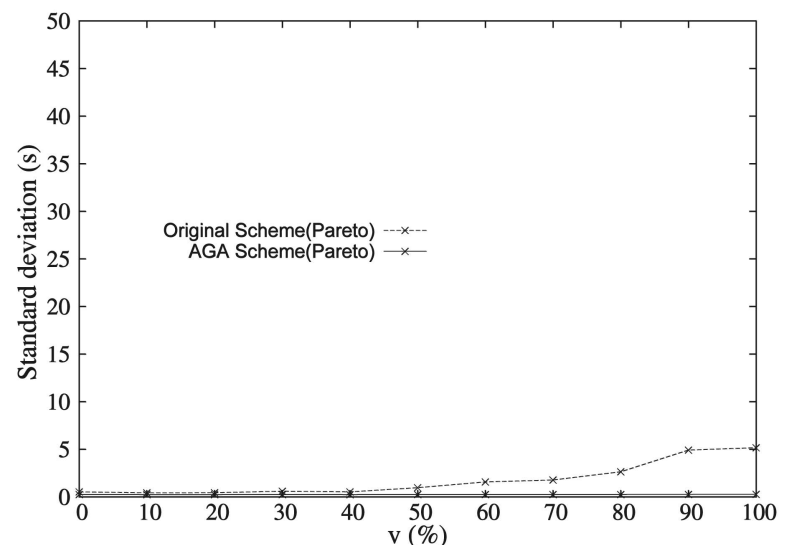

(b)

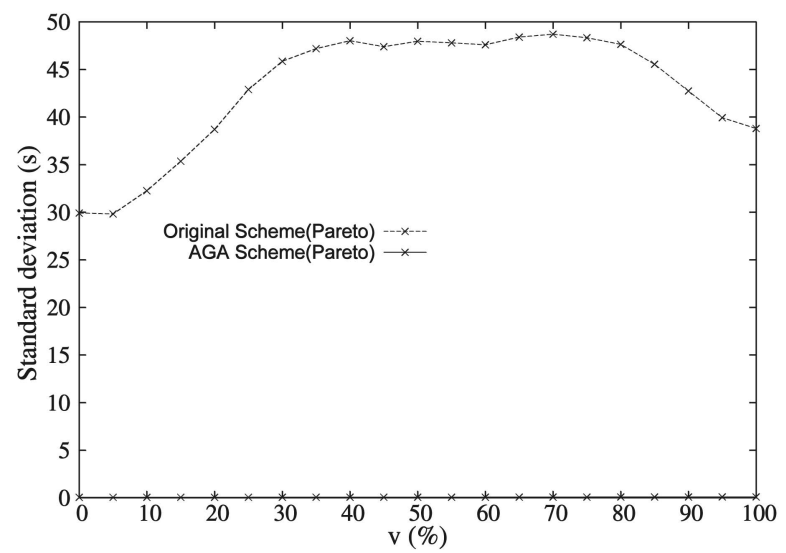

(d)

Fig. 7. Effect of traffic load on standard deviation $\sigma$ (in seconds). (a) $N=10$. (b) $N=10$. (c) $N=20$. (d) $N=20$.

can receive the service without any modification. The performance evaluation for our AGA scheme was conducted through mathematical analysis and simulation experiments. The mathematical analysis was developed based on a Markov-Chain-like methodology, where the transition probabilities of the formulated Markov Chain are not constants, and the steady states cannot be obtained through traditional probabilistic derivations. The capability of the proposed AGA scheme was also evaluated by a series of simulation experiments. Some practical traffic models such as the Gamma and Pareto distributions with self similarity were adopted for the experiments. The numerical results indicate that our proposed scheme greatly outperforms the existing IEEE 802.15.4 implementations.

\section{ACKNOWLEDGMENTS}

This work was supported in part by the Excellent Research Projects, National Taiwan University, under Contract 96R0062-AE00-07 and the National Science Council under Contracts NSC95-2221-E-002-096-MY3，96-2219-E-002-004, 94-2118-M-009-003, and III.

\section{REFERENCES}

[1] Zigbee Alliance, http://www.zigbee.org, 2007.

[2] IEEE Standard for Information Technology Part 15.3: Wireless Medium Access Control (MAC) and Physical Layer (PHY) Specifications for Wireless Personal Area Networks (WPANs), IEEE Standard 802.15.3 Working Group Std., 2003.
[3] IEEE Standard for Information Technology Part 15.4: Wireless Medium Access Control (MAC) and Physical Layer (PHY) Specifications for Low-Rate Wireless Personal Area Networks (LR-WPANs), IEEE Standard 802.15.4 Working Group Std., 2003.

[4] E. Callaway, P. Gorday, and L. Hester, "Home Networking with IEEE 802.15.4: A Developing Standard for Low-Rate Wireless Personal Area Networks," IEEE Comm. Magazine, Aug. 2002.

[5] M. Crovella and A. Bestavros, "Self-Similarity in World Wide Web Traffic: Evidence and Possible Causes," IEEE/ACM Trans. Networking, Dec. 1997.

[6] X. Dong, P. Varaiya, and A. Puri, "Adaptive Polling Algorithm for PCF Mode of IEEE 802.11 Wireless LANs," Electronics Letters, Apr. 2004.

[7] J.A. Gutierrez, "On the Use of IEEE 802.15.4 to Enable Wireless Sensor Networks in Building Automation," Proc. 15th IEEE Int'l Symp. Personal, Indoor and Mobile Radio Comm. (PIMRC '04), Sept. 2004.

[8] R.K. Jain, D.W. Chiu, and W.R. Hawe, "A Quantitative Measure of Fairness and Discrimination for Resource Allocation in Shared Computer Systems," DEC Technical Report TR-301, Sept. 1984.

[9] A. Koubaa, M. Alves, and E. Tovar, "i-GAME: An Implicit GTS Allocation Mechanism in IEEE 802.15.4 for Time-Sensitive Wireless Sensor Networks," Proc. 18th Euromicro Conf. Real-Time Systems (ECRTS '06), July 2006.

[10] J.F. Kurose and K.W. Ross, Computer Networks. Addison Wesley, 2003.

[11] N.L. Johnson, Discrete Distributions. Houghton Mifflin, 1969.

[12] Q. Liu, X. Wang, and G.B. Giannakis, "A Cross-Layer Scheduling Algorithm with QoS Support in Wireless Networks," IEEE Trans. Vehicular Technology, May 2006.

[13] G. Lu, B. Krishnamachari, and C.S. Raghavendra, "Performance Evaluation of the IEEE 802.15.4 MAC for Low-Rate Low-Power Wireless Networks," Proc. 23rd IEEE Int'l Performance, Computing and Comm. Conf. (IPCCC '04), Apr. 2004. 
[14] J. Ma, M. Gao, Q. Zhang, L.M. Ni, and W. Zhu, "Localized LowPower Topology Control Algorithms in IEEE-802.15.4-Based Sensor Networks," Proc. 25th IEEE Int'l Conf. Distributed Computing Systems (ICDCS '05), June 2005.

[15] J. Mišić, S. Shaf, and V.B. Mišić, "Performance of a Beacon Enabled IEEE 802.15.4 Cluster with Downlink and Uplink Traffic," IEEE Trans. Parallel and Distributed Systems, vol. 17, no. 4, pp. 361-376, Apr. 2006.

[16] D.A. Patterson and J.L. Hennessy, Computer Organization and Design. Morgan Kaufmann, 1997.

[17] Bluetooth Specification. Bluetooth SIG, 1999.

[18] N.F. Timmons and W.G. Scanlon, "Analysis of the Performance of IEEE 802.15.4 for Medical Sensor Body Area Networking," Proc. First Ann. IEEE Conf. Sensor and Ad Hoc Comm. and Networks (SECON '04), Oct. 2004.

[19] Y.-C. Tseng and M.-S. Pan, "Quick Convergecast in Zigbee/IEEE 802.15.4 Tree-Based Wireless Sensor Networks," Proc. Fourth Int'l Workshop Mobility Management and Wireless Access (MobiWac '06), Oct. 2006.

[20] J.-S. Yang, C.-C. Tseng, and R.-G. Cheng, "Dynamic Scheduling Framework on an RLC/MAC Layer for General Packet Radio Service," IEEE Trans. Wireless Comm., Sept. 2003.

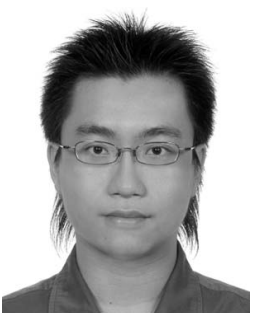

Yu-Kai Huang received the BS degree from the National Tsing Hua University, Taiwan, in 2003 and the MS degree from the National Taiwan University, Taiwan, in 2005. He is currently working toward the $\mathrm{PhD}$ degree in the Graduate Institute of Networking and Multimedia, Department of Computer Science and Information Engineering, National Taiwan University. His research interests are focused on low-rate wireless personal area networks, especially resource management and energy-efficient issues. He is a student member of the IEEE.

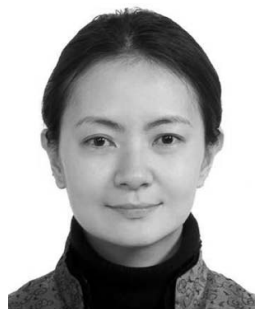

Ai-Chun Pang received the BS, MS, and $\mathrm{PhD}$ degrees in computer science and information engineering from the National Chiao Tung University, Taiwan, in 1996, 1998, and 2002, respectively. She joined the Department of Computer Science and Information Engineering, National Taiwan University (CSIE NTU), Taipei, as an assistant professor in 2002. From August 2004 to July 2005, she was an assistant professor in the Graduate Institute of Networking and Multimedia (INM) and an adjunct assistant professor in CSIE, NTU, where she is currently an associate professor. Her research interests include the design and analysis of personal communications services network, mobile computing, voice over IP, and performance modeling. She was a program cochair of many international conferences/workshops. She was the guest editor of the IEEE Wireless Communications and is currently an associate editor for the International Journal of Sensor Networks. She received the Teaching Award from NTU in 2005, 2006, and 2007 and the Investigative Research Award from the Pan Wen Yuan Foundation in 2006. She is a member of the IEEE.

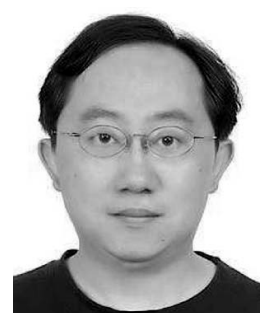

Hui-Nien Hung received the $\mathrm{PhD}$ degree in statistics from the University of Chicago, Chicago, Illinois in 1996. He is currently a professor in the Institute of Statistics, National Chiao Tung University, Hsinchu, Taiwan. His research interests include applied probability, biostatistics, statistical inference, statistical computing, and industrial statistics.

$\triangleright$ For more information on this or any other computing topic, please visit our Digital Library at www.computer.org/publications/dlib. 